\title{
Recurring obscuration in NGC 3783
}

\author{
J. S. Kaastra ${ }^{1,2}$, M. Mehdipour ${ }^{1}$, E. Behar ${ }^{3}$, S. Bianchi ${ }^{4}$, G. Branduardi-Raymont ${ }^{5}$, L. Brenneman ${ }^{6}$, M. Cappi ${ }^{7}$, \\ E. Costantini ${ }^{1}$, B. De $\mathrm{Marco}^{8}$, L. di Gesu ${ }^{9}$, J. Ebrero ${ }^{10}$, G. A. Kriss ${ }^{11}$, J. Mao ${ }^{1,2}$, U. Peretz ${ }^{3}$, P.-O. Petrucci ${ }^{12}$, \\ G. Ponti ${ }^{13}$, and D. Walton ${ }^{14}$
}

${ }^{1}$ SRON Netherlands Institute for Space Research, Sorbonnelaan 2, 3584 CA Utrecht, The Netherlands e-mail: J.S.Kaastra@sron.nl

2 Leiden Observatory, Leiden University, PO Box 9513, 2300 RA Leiden, The Netherlands

3 Department of Physics, Technion-Israel Institute of Technology, Haifa 32000, Israel

${ }^{4}$ Dipartimento di Matematica e Fisica, Università degli Studi Roma Tre, Via della Vasca Navale 84, 00146 Roma, Italy

5 Mullard Space Science Laboratory, University College London, Holmbury St. Mary, Dorking, Surrey, RH5 6NT, UK

6 Smithsonian Astrophysical Observatory, Cambridge, MA, USA

7 INAF-IASF Bologna, via Gobetti 101, 40129 Bologna, Italy

${ }^{8}$ Nicolaus Copernicus Astronomical Center, Polish Academy of Sciences, Bartycka 18, 00-716 Warsaw, Poland

9 Department of Astronomy, University of Geneva, 16 Ch. d'Ecogia, 1290 Versoix, Switzerland

${ }_{10}$ European Space Astronomy Centre, PO Box 78, 28691 Villanueva de la Cañada, Madrid, Spain

11 Space Telescope Science Institute, 3700 San Martin Drive, Baltimore, MD 21218, USA

12 Univ. Grenoble Alpes, CNRS, IPAG, 38000 Grenoble, France

13 Max Planck Institute für Extraterrestriche Physik, 85748 Garching, Germany

14 Institute of Astronomy, Madingley Road, CB3 0HA Cambridge, UK

Received 12 January 2018 / Accepted 25 August 2018

\begin{abstract}
Context. Obscuration of the continuum emission from active galactic nuclei by streams of gas with relatively high velocity $\left(>1000 \mathrm{~km} \mathrm{~s}^{-1}\right)$ and column density $\left(>3 \times 10^{25} \mathrm{~m}^{-2}\right)$ has been seen in a few Seyfert galaxies. This obscuration has a transient nature. In December 2016 we witnessed such an event in NGC 3783.

Aims. The frequency and duration of these obscuration events is poorly known. Here we study archival data of NGC 3783 in order to constrain this duty cycle.

Methods. We use archival Chandra/NuSTAR spectra taken in August 2016. We also study the hardness ratio of all Swift XRT spectra taken between 2008 and 2017.

Results. In August 2016, NGC 3783 also showed evidence of obscuration. While the column density of the obscuring material is ten times lower than in December 2016, the opacity is still sufficient to block a significant fraction of the ionising X-ray and extreme ultraviolet photons. From the Swift hardness ratio behaviour we find several other epochs with obscuration. Obscuration with columns $>10^{26} \mathrm{~m}^{-2}$ may take place about half of the time. Also, in archival X-ray data taken by the Advanced Satellite for Cosmology and Astrophysics (ASCA) in 1993 and 1996 we find evidence of obscuration.

Conclusions. Obscuration of the ionising photons in NGC 3783 occurs more frequently than previously thought. This may not always have been recognised due to low-spectral-resolution observations, overly limited spectral bandwidth or confusion with underlying continuum variations.
\end{abstract}

Key words. X-rays: galaxies - galaxies: active - galaxies: Seyfert - galaxies: individual: NGC 3783 - techniques: spectroscopic

\section{Introduction}

Outflows from active galactic nuclei (AGN) have been found in about half of all nearby systems (Crenshaw et al. 2003). They become visible because of the imprint of absorption lines and edges in the X-ray and ultraviolet (UV) spectra of these objects. They show a broad range of ionisation states with column densities usually up to about $10^{26} \mathrm{~m}^{-2}$ at the highest ionisation parameters. These outflows may originate from an accretion disk wind or torus wind, and have speeds up to a few thousand kilometers per second or even much higher if ultra-fast outflows are considered. But all these outflow components share the property that the total flux taken away from the continuum radiation is modest.

There is also evidence for outflows with much higher column densities and often at lower ionisation states than those described above, which absorb so much soft X-ray and extreme ultraviolet (EUV) radiation that we may call them obscurers. While in individual cases X-ray obscuration has been demonstrated to be present in charge-coupled device (CCD) spectra, the relatively low resolution of CCDs prevents investigation of the dynamics of these components. Examples for the latter are the X-ray eclipses in Mrk 766 and NGC 1365 that were attributed to broad line region clouds passing our line of sight (Risaliti et al. 2007, 2011).

This situation changed when NGC 5548 was found to be obscured in 2013 (Kaastra et al. 2014b). It was observed simultaneously with high-resolution X-ray detectors (the Reflection Grating Spectrometer (RGS) on XMM-Newton), broadband X-ray instruments (European Photon Imaging Camera (EPIC) on XMM-Newton, Nuclear Spectroscopic Telescope Array $(N u S T A R)$ ), high-resolution UV spectroscopy (Cosmic 
Origins Spectrograph (COS) on the Hubble Space Telescope (HST)), and photometry in the optical/UV band (the Optical Monitor (OM) on XMM-Newton, the Ultraviolet/Optical Telescope (UVOT) on Swift). The X-ray data revealed two obscuring components with column densities of $10^{26}-10^{27} \mathrm{~m}^{-2}$ and $\mathrm{X}$-ray covering factors of $0.86-0.30$ that were found to range from lowly ionised to almost neutral for both components, respectively. The low ionisation component produces broad UV absorption lines which reveal that the obscuring material is outflowing with velocities up to $5000 \mathrm{~km} \mathrm{~s}^{-1}$. In general, the UV covering factor is about three times lower than the X-ray covering factor, and the obscuring material is likely to be close to the outer broad line region. Two further sources showing signs of obscuration and studied with joint UV and X-ray spectroscopy, although with much sparser observations, are Mrk 335 (Longinotti et al. 2013) and NGC 985 (Ebrero et al. 2016).

Triggered by these results on NGC 5548, we have started a monitoring program with Swift on a sample of Seyfert galaxies to find them in obscured states using the hardness ratio of the $\mathrm{X}$-ray spectra as a discerning diagnostic against simple flux variability. In an obscured state, the spectrum significantly hardens. This program triggered successfully on NGC 3783 in December 2016 (Mehdipour et al. 2017). Simultaneous observations with XMM-Newton, HST, and NuSTAR showed that NGC 3783 was in a similar obscured state to NGC 5548. However, while the obscuration of NGC 5548 has already been going on for several years, the event in NGC 3783 lasted for about a month, as evidenced by the Swift monitoring.

In order to better understand the obscuration phenomenon, it is important to know its duty cycle: how frequent are these events, and what is their duration. NGC 3783 was selected for our monitoring program because previous monitoring with the Rossi X-ray Timing Explorer (RXTE) has shown one obscuring event in March 2008 and two possible events in July 2008 and February 2011 (Markowitz et al. 2014). Swift, with its softer energy band as compared with RXTE, is even more sensitive to these events, and NGC 3783 was monitored intensively with Swift in Spring 2017, after the obscuration event in December 2016 described by Mehdipour et al. (2017). Furthermore, in August 2016, coordinated Chandra High-Energy Transmission Grating Spectrometer (HETGS) and NuSTAR observations were performed on NGC 3783. Finally, Advanced Satellite for Cosmology and Astrophysics (ASCA) data taken in 1993 showed remarkable differences compared with spectra taken three years later (George et al. 1998). In this paper we re-analyse these data in order to better constrain the duty cycle of the obscuration in NGC 3783.

\section{Data analysis}

\subsection{Data reduction}

There are two Chandra/NuSTAR observations separated by three days taken in August 2016 (PI: L Brenneman). The data used for our spectral analysis are shown in Table 1.

We used the Chandra HETGS spectra from the tgcat archive (Huenemoerder et al. 2011). The spectra were rebinned by a factor of two to approximate the optimal binning of the data (Kaastra \& Bleeker 2016). We restricted our fits to the 2.5-26 $\AA$ range for the Medium Energy Grating (MEG), and 1.55-14.5 $\AA$ for the High Energy Grating (HEG). We renormalise the HEG data relative to the MEG data adopting a scaling factor of 0.954, equal to what we used for Mrk 509 (Kaastra et al. 2014a).
The NUSTAR data were taken from the public archive and extracted using the standard tools. Spectra of both detectors were combined into a single spectrum using the HEAsoft addspec tool. We restricted our fits to the 4-79 keV range. We renormalised the $N u S T A R$ data relative to the MEG by a factor of $0.849 \pm 0.018$, determined from comparing the fluxes of both instruments in the 4.5-6.0 keV band. This renormalisation incorporates both calibration uncertainties as well as possible small differences in the spectra because of slightly different time intervals (Table 1).

Swift UVOT data, taken simultaneously with the first Chandra/NuSTAR observation, were extracted as described in Mehdipour et al. (2017). Lacking UVOT data for the second observation, we reused the fluxes from the first observation. Due to the short exposure time compared to the other X-ray instruments we did not use the Swift X-Ray Telescope (XRT) data for our spectral analysis.

Archival ASCA spectra were taken from the Tartarus database (Turner et al. 2001). This database also contains the response matrices and background spectra. ASCA observed NGC 3783 twice in 1993, four times in 1996, and three times in 2000. The observations in each year were taken within the same week and we have combined the spectra into a single spectrum for each year. Although there are moderate differences between the spectra within one week, the differences between the average 1993 and 1996 spectra are much larger (George et al. 1998). In this paper we focus on these time-averaged ASCA spectra.

We also combined the Solid-state Imaging Spectrometers (SIS0 and SIS1) data. This data addition was performed using the Heasoft addspec utility. The 1993 and 1996 spectra show a strong spectral difference at low energies (George et al. 1998). A plot of all three spectra shows that the spectrum in 2000 is very similar to that from 1996, except for a $\sim 30 \%$ weaker soft excess at $0.5 \mathrm{keV}$ (Fig. 3). Given their higher spectral resolution, here we use data taken with SIS0 and SIS1 detectors and ignore the Gas Imaging Spectrometers (GIS2 and GIS3) data.

Lacking contemporaneous UV data for the ASCA epochs, we have assumed that the UVOT fluxes as observed in August 2016 were the same as in 1993 and 1996. While in reality there may be a significant difference, this choice prevents the Comptonised soft excess component from becoming unrealistically unconstrained due to extrapolation from the X-ray band. Therefore we fit the ASCA spectra together with the UVOT data, but we only report the goodness of fit for the ASCA part of the data.

The properties of the obscurer do not depend significantly on the precise UV flux level. For instance, lowering the UV flux by a factor of two for the 1993 ASCA data affects the parameters of the comptonised soft excess, but the column density and covering factor of the obscurer remain unchanged within their statistical uncertainties.

\subsection{Spectral modelling}

All spectral modelling was done using the SPEX package (Kaastra et al. 1996). The basic spectral model is summarised here in some detail. It is the model used by Mehdipour et al. (2017) and that paper justifies the choice for the model and the adopted parameters.

The spectral model is described by the following three components (the SPEX names of the components are in italics):

1) Power law (pow) with exponential cut-off (etau) at high energies $(340 \mathrm{keV})$ and low energies $(1.53 \mathrm{eV})$ representing the dominant emission component.

2) Comptonised soft X-ray excess (comt).

3) Eight X-ray broad lines, modelled as delta lines with Gaussian 
Table 1. Chandra HETGS, NuSTAR and Swift UVOT spectral data used in this study.

\begin{tabular}{lcccc}
\hline \hline Start date and time & End date and time & ObsID & Instrument & Net exposure time (ks) \\
\hline $22-8-201607: 24$ & $23-8-201606: 10$ & 18192 & HETGS & 79 \\
$22-8-201606: 26$ & $23-8-201606: 21$ & 60101110002 & NuSTAR & 41 \\
$22-8-2016$ 09:15 & $22-8-201609: 29$ & 81760001 & UVOT & 2 \\
$25-8-201600: 23$ & $25-8-201621: 30$ & 19694 & HETGS & 73 \\
$24-8-201621: 16$ & $25-8-201621: 56$ & 60101110004 & NuSTAR & 42 \\
\hline
\end{tabular}

Table 2. Best-fit parameters derived in this work for the August 2016 spectra of NGC 3783 and the ASCA spectra taken in 1993, 1996 , and 2000.

\begin{tabular}{|c|c|c|c|c|c|c|c|}
\hline Date & $12-1993$ & $7-1996$ & $1-2000$ & $22-8-2016$ & $25-8-2016$ & $11-12-2016$ & 21-12-2016 \\
\hline \multicolumn{8}{|l|}{ Component 1} \\
\hline$N_{\mathrm{H}}\left(10^{27} \mathrm{~m}^{-2}\right)$ & - & - & - & - & - & $1.5 \pm 0.2$ & $2.0 \pm 0.2$ \\
\hline$C_{\mathrm{f}}$ & - & - & - & - & - & $0.47 \pm 0.10$ & $0.38 \pm 0.03$ \\
\hline \multicolumn{8}{|l|}{ Component 2} \\
\hline$N_{\mathrm{H}}\left(10^{27} \mathrm{~m}^{-2}\right)$ & $0.17 \pm 0.02$ & $0.11 \pm 0.02$ & $0.036 \pm 0.003$ & $0.18 \pm 0.02$ & $0.44 \pm 0.06$ & $0.8 \pm 0.2$ & $0.3 \pm 0.1$ \\
\hline$C_{\mathrm{f}}$ & $0.69 \pm 0.02$ & $0.27 \pm 0.03$ & $1.00 \pm 0.05$ & $0.63 \pm 0.05$ & $0.45 \pm 0.02$ & $0.51 \pm 0.10$ & $0.48 \pm 0.03$ \\
\hline \multicolumn{8}{|l|}{ Power law } \\
\hline$\Gamma$ & 1.91 & 1.78 & 1.86 & 1.81 & 1.78 & 1.71 & 1.75 \\
\hline$L_{2-10 \mathrm{keV}}\left(10^{36} \mathrm{~W}\right)$ & 2.56 & 3.17 & 3.21 & 1.31 & 1.10 & 0.95 & 1.16 \\
\hline \multicolumn{8}{|l|}{ Comptonised disk } \\
\hline$L\left(10^{36} \mathrm{~W}\right)$ & 20.3 & 8.7 & 7.6 & 11.8 & 8.0 & 15.7 & 17.7 \\
\hline$T_{0}(\mathrm{eV})$ & 2.3 & 1.3 & 1.5 & 1.5 & 1.3 & 1.1 & 1.1 \\
\hline$T_{1}(\mathrm{eV})$ & 142 & 141 & 132 & 145 & 129 & 128 & 131 \\
\hline \multicolumn{8}{|l|}{ Goodness of fit } \\
\hline$C$-stat & 539 & 520 & 283 & 5077 & 4721 & 2288 & 2285 \\
\hline Expected $C$-stat & 228 & 184 & 140 & 4706 & 4497 & 1539 & 1542 \\
\hline
\end{tabular}

Notes. For comparison we also list the best-fit parameters as obtained by Mehdipour et al. (2017) for the December 2016 spectra.

velocity broadening (delt with vgau applied), for $\mathrm{O}$ VIII and $\mathrm{N}$ vII Ly $\alpha$ and the He-like triplets of O VII and N vI.

These emission components are then fed successively through the obscurer (modelled by two xabs components and one pion component for the high-ionisation component) and the nine warm absorber components (all modelled with the pion component, accounting only for their absorption properties). We note that for each of these successive absorption steps the ionising spectrum becomes weaker. The finally transmitted spectrum is then cosmologically redshifted and corrected for absorption in our Galaxy. This Galactic absorption is done using the hot model for the X-ray part of the spectrum $(E>13.6 \mathrm{eV})$ and the $\mathrm{ebv}$ model for the optical/UV part. Technically, this is achieved by duplicating these components and by deleting either the UV part or the X-ray part with an etau component with large, constant optical depth over the relevant part of the spectrum.

Further we have three other emission components:

4) Power law (pow) with exponential cut-off (etau) at high energies $(340 \mathrm{keV})$ and low energies $(1.2 \mathrm{eV})$.

5) Comptonised soft excess (comt).

6) Disc reflection component ( $r e f$ ) plus a narrow Gaussian line (to represent the $\mathrm{Fe} \mathrm{K} \beta$ line which is not included in the refl model) from distant reflecting material.

These three emission components (4-6) represent the longterm time-average ionising continuum for the distant X-ray narrow line emission region. They are fed through the two pion emission components representing the narrow line emission region, and then we delete the flux of components 4-5 by applying again an etau component with high optical depth, in order not to duplicate the actually seen continuum already accounted for by components 1 and 2 . The reflection component is new here and accounted for only in this part of the calculation.

Finally, we add two constant emission terms:

7) The host galaxy optical/UV continuum in the spectral extraction region.

8) The optical emission line and continuum spectral features.

Both of these latter two components are represented with a file component, and they are described in more detail by Mehdipour et al. (2017).

The flux of NGC 3783 in the soft band was relatively low during these observations, and the effective area of the HETGS below $1 \mathrm{keV}$ has decreased significantly over its lifetime. Therefore the signal to noise ratio $(\mathrm{S} / \mathrm{N})$ of the HETGS data at lower energies is not optimal. This means we have to constrain the number of free parameters.

We use, as the baseline, the model parameters for the obscured spectrum as taken on December 11, 2016 by XMM-Newton, NuSTAR and HST-COS. This model has been described in detail by Mehdipour et al. (2017). Briefly, Mehdipour et al. (2017) first determine the properties of the outflow ("warm absorber") from archival XMM-Newton and Chandra HETGS spectra obtained between 2000 and 2001 and in 2013. The outflow has nine components, all modelled with the pion model of SPEX, and with different combinations of ionisation parameters and outflow velocity. For the obscured state observed in December 2016 they then assume that all parameters of the outflow components remain the same except for the ionisation parameters, which are calculated (not fitted) from the 

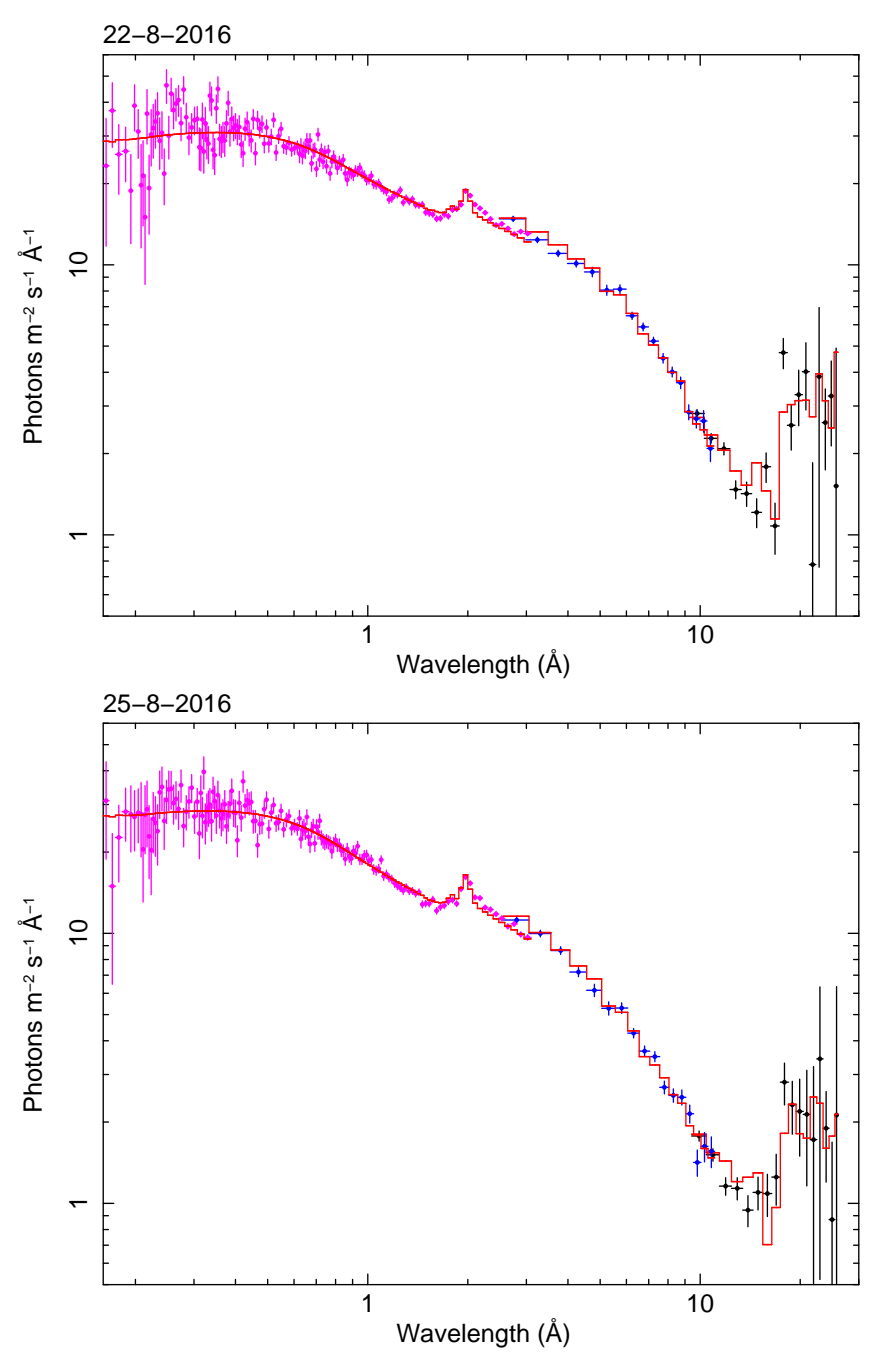

Fig. 1. Fluxed spectra of NGC 3783 on August 22 and August 25, 2016, using the model described in the text. Red curve: best-fit model; purple crosses: NUSTAR data; blue and black crosses: HEG and MEG data, both rebinned by a factor of 100 for display purposes.

ionising continuum that the outflow receives assuming that the gas density and distance to the ionising source have remained constant between 2001 and 2016. This ionising continuum is determined from fitting the observed spectrum with the primary continuum parameters (power law, reflection component and comptonised soft excess) and intervening obscurer parameters left free and including the outflow as described above.

In addition to the primary continuum, affected by both the obscuring medium and the warm absorber, the Mehdipour et al. (2017) model also contains some emission from gas far away from the nucleus which produces narrow emission features.

In our model of the present August 2016 data we freeze the parameters of these narrow emission components to the ones found in the spectra from December 2016. The present HETGS data are not extremely sensitive to these emission features, so this choice does not affect our final model. Again related to sensitivity issues, we keep the reflection component constant to the December values, and also the optical depth of the comptonised soft excess is kept constant. The only free parameters of our model are therefore the normalisation, photon index and exponential cut-off of the primary power-law component, the normalisation, seed photon temperature $T_{0}$ and electron temperature $T_{1}$ of the comptonised soft excess, and the column densities and covering factors of the obscuring gas. All other parameters are frozen to the December 2016 values or calculated assuming that $n r^{2}=L / \xi$ is constant for each component, with $n, r, L$, and $\xi$ being the hydrogen density, distance to the ionising source, ionising luminosity, and ionisation parameter, respectively.

For the December 2016 spectrum, the presence or absence of specific ions in the high-resolution UV spectra taken simultaneously with HST/COS allowed us to constrain the ionisation parameter of the obscurer. In the present case, there are no simultaneous high-resolution UV spectra. Therefore, for the obscurer we adopt the same ionisation parameters as in December 2016, which is also justified because the X-ray transmission of the obscurer is not very sensitive to this ionisation parameter.

All fits were done using the full set of X-ray and UV spectra. Because we lack simultaneous UV data for all but one epoch, the UV spectra merely serve to constrain the spectral model within realistic limits, as discussed before. Due to UV variability (see for instance Fig. 4) the actual UV flux may differ from our adopted values. We have tested that a factor of two higher or lower UV flux does not affect the derived fit parameters as listed in Table 2 in a substantial way. In general, the UV flux points match the best-fit models within $20 \%$.

For the spectral fitting, we use the $C$-statistics as implemented in SPEX (Kaastra 2017). The best-fit parameters are shown in Table 2. We find that one obscuring component is sufficient to model all these data. Figure 1 shows how well our model matches the broad-band spectrum in August 2016, and Fig. 2 shows the corresponding Chandra spectra in greater detail in the 4.5-11 $\AA$ band, where the signal is strong enough to reveal individual absorption lines. Figure 3 shows the best fit for the ASCA data.

\subsection{Swift light curves}

Figure 4 shows the Swift light curves in different bands as well as the X-ray hardness ratio $R$, which provides an indication for obscuration when it is high enough. The hardness is defined by $R=(H-S) /(H+S)$ with $S$ and $H$ being the soft and hard X-ray count rates in the $0.3-1.5$ and $1.5-10 \mathrm{keV}$ bands, respectively. The model of Mehdipour et al. (2017) for the unobscured spectrum based on all archival Chandra HETGS and XMM-Newton data taken in 2000 and 2001 yields a predicted hardness ratio $R=0.07$. In that model, the photon index of the primary power law is 1.60. Making the spectrum harder, with a photon index of 1.40 , increases $R$ to 0.15 . Alternatively, removing completely the soft excess also raises $R$ to a value of 0.15 . We therefore adopt here a range of -0.01 to +0.15 as the fiducial hardness range corresponding to the natural variations of the primary AGN continuum.

Using the same parameters for the unobscured spectrum based on the 2000 and 2001 spectra, we predict the hardness $R$ in case there is additional obscuration like that modelled for the August 2016 Chandra spectra and the ASCA spectra. Our results are shown in Fig. 5. For zero covering factor, the hardness ratio is 0.07 . For larger covering factors, $R$ increases with increasing column density. It reaches a maximum for column densities in the range $3-5 \times 10^{26} \mathrm{~m}^{-2}$ and then declines. At high column densities, the full covered primary continuum below $10 \mathrm{keV}$ gets blocked. In that case, only the distant, uncovered, and constant reflection component of our model remains present, plus the uncovered fraction of the primary continuum. This explains the decline of the hardness ratio at high column densities. 

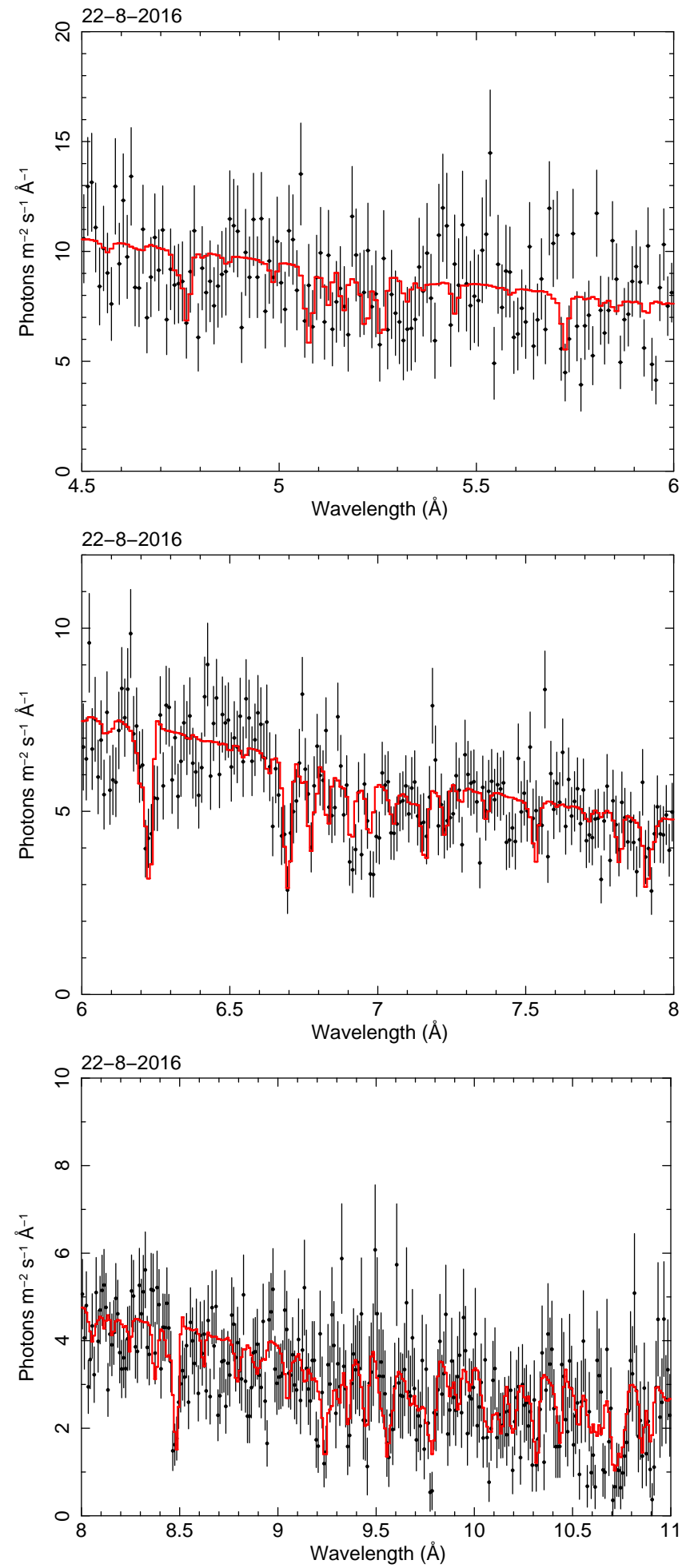
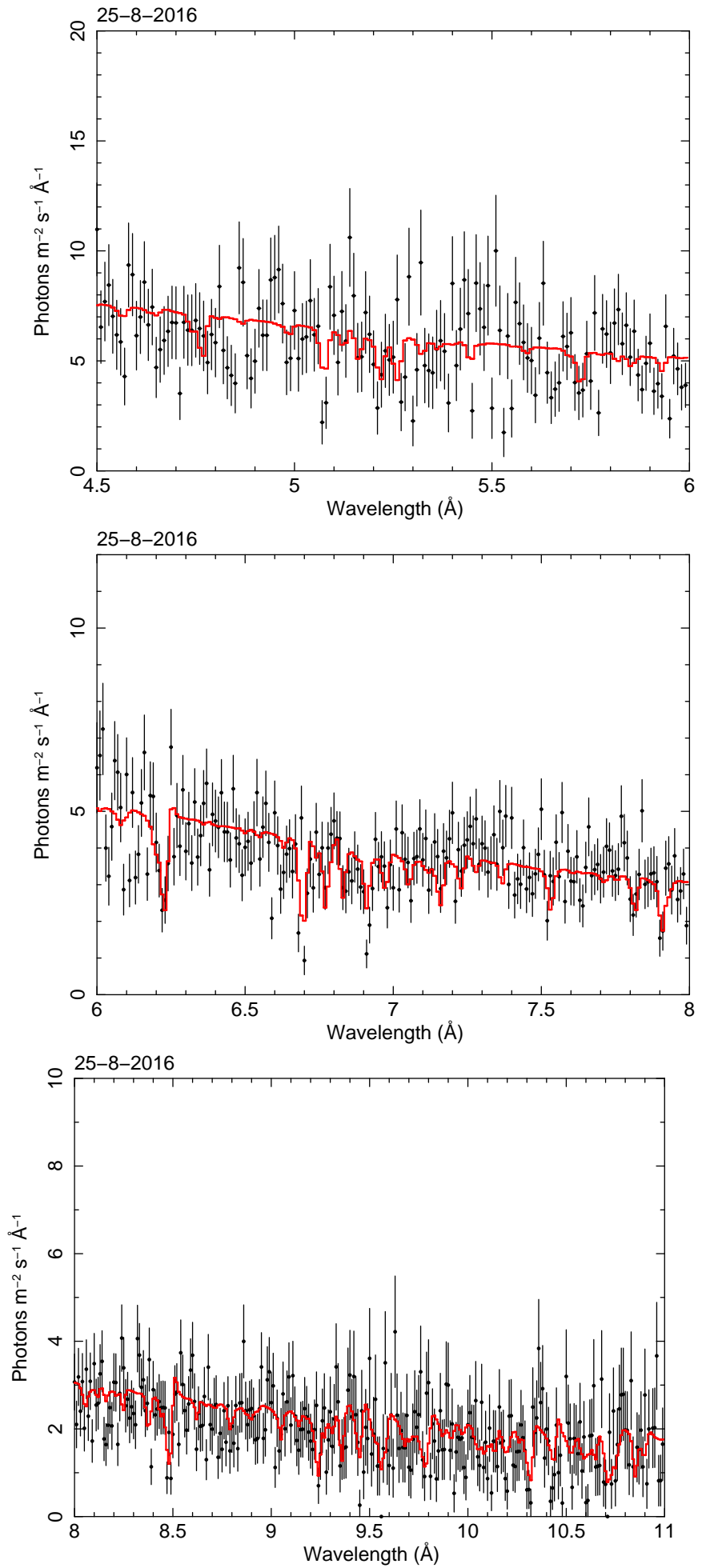

Fig. 2. Fluxed spectra of NGC 3783 on August 22, 2016 (left) and August 25, 2016 (right) using the model described in the text. Red curve: best-fit model; black crosses: MEG data.

The maximum value that $R$ can achieve is about 0.8 for full covering and a column density of about $5 \times 10^{26} \mathrm{~m}^{-2}$. Indeed Fig. 4 shows no epochs with $R$ above this value.

In Fig. 4 we have indicated the level of $R=0.27$ by a dashed horizontal line. This value corresponds to the maximum hardness for covering factor 0.5 . Values $R>0.27$ correspond to covering factors of at least $50 \%$ and column densities larger than $5 \times 10^{25} \mathrm{~m}^{-2}$. We may call these strongly obscured states.
We note that the flux decrease due to the obscuration is enhanced by the distant warm absorber: it receives less ionising radiation, recombines and achieves higher opacity. Further, the model for $R$ presented in Fig. 5 only holds for a single obscuration component. In case of multiple components, like in December 2016, the situation is more complex because there are more free parameters. In that case, however, the flux decrease is even stronger due to the combined effect of all components. 


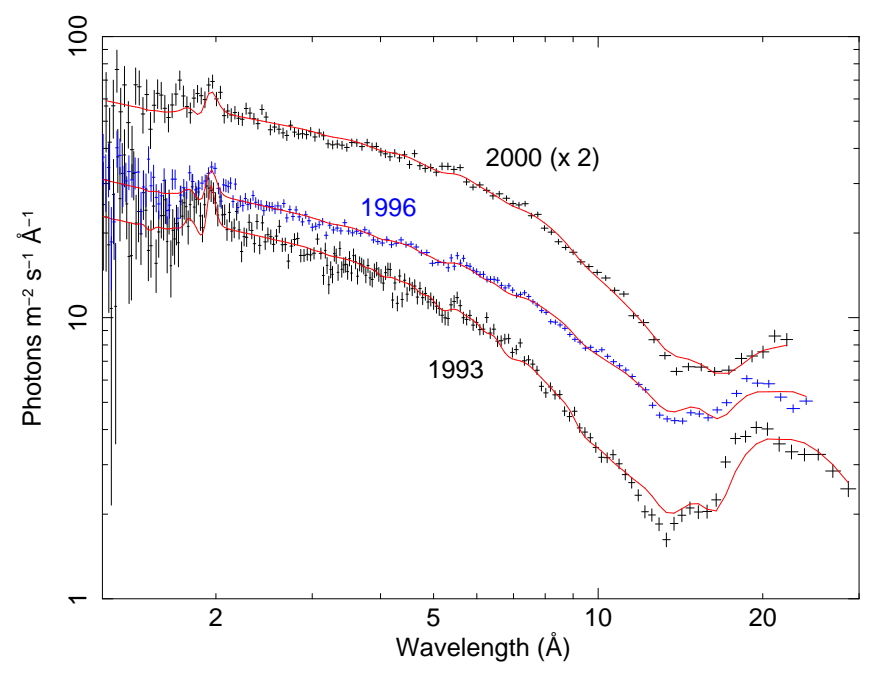

Fig. 3. ASCA SIS spectra taken in 1993 (black crosses, lower spectrum), 1996 (blue crosses, middle spectrum) and 2000 (black crosses, upper spectrum, for display purposes scaled by a factor of two), together with the best-fit models as shown by the red lines. See Sect. 3.2 for an explanation of the deviations at long wavelengths.

There are several epochs where the hardness is large and strong obscuration is present. One event occurred in 2009, but with the sparse sampling in that year it is hard to put that into context. The second event is seen in December 2016 (days 210-250 in Fig. 4) and is discussed in detail by Mehdipour et al. (2017). From mid-January 2017 (day 270) the hardness increased gradually over two weeks time to reach a similar level as in December 2016. It remained high for about two months, with substantial variation. The latest data in autumn 2017, taken after a data gap in summer, show again such a hard state.

Interestingly, in August 2016 when the Chandra data were taken (day 122 in Fig. 4), the Swift hardness ratio was also high (0.35) but below the trigger level for the XMM-Newton program. As we have shown, in this state there is also obscuration but less than in December 2016. The Swift hardness ratio curve shows several other epochs with similar hardness, like in 2014 and March 2016. In fact, $66 \%$ of all Swift data points show a hardness larger than 0.27 , although we must take care because the spacing of the data points is not even.

\section{Discussion}

\subsection{Obscuration in August 2016}

The spectral fits that we have obtained can be characterised by the deep broad dip beyond about $12 \AA$ that reaches a minimum near 15-17 $\AA$ (Fig. 1). This is caused by a deep (optical depth 1.7) $\mathrm{O}$ vII edge produced by the obscuring material, and enhanced by a multitude of iron lines from the warm absorber. Such a deep edge is not present in the normal, unobscured spectrum of NGC 3783 .

Our fit reproduces the main features of the present NGC 3783 spectra well, as can be seen from both the rebinned broad-band spectra (Fig. 1) and the detailed HETGS spectra showing good matches of the strongest absorption lines with the model (Fig. 2).

The ionising continuum in August 2016 is slightly different from the continuum four months later. While the luminosity of the power law component is similar for both epochs, the comptonised soft excess is about $40 \%$ weaker in August, which can also be seen more directly from the lower flux observed with the Swift UVOT.

Interestingly, NGC 3783 shows clear evidence for obscuration in August 2016, although lower than four months later in December. While the covering factor of the obscurer is of the same order of magnitude or even slightly larger than in December, the column density is an order of magnitude lower, but still sufficient to remove a significant fraction of the X-ray flux. We show the transmission profile of the obscurer in Fig. 6. Because of the partial covering, $37 \%$ of the X-ray flux is always transmitted. It is seen from this figure that the transmission curve shows a lot of structure. At the shortest wavelengths, the transmission drops gradually from unity to close to the residual level of $37 \%$, with a few broadened absorption lines superimposed. In the $15-100 \AA$ band, there is detailed structure with a combination of deep edges from oxygen ions and strong absorption lines. But also the opacity in the EUV band up to the Lyman edge is structured, and a significant fraction of the ionising EUV continuum is absorbed.

It should be noted that, like in other cases, the obscuring material has a strong recombining effect on the warm absorber. The total X-ray blocking from the combined obscurer and recombined warm absorber is much stronger than during unobscured phases like those seen in 2000 and 2001.

Our model predicts that the $2 \mathrm{~s}-2 \mathrm{p}$ transitions of $\mathrm{C}$ IV lines at 1548 and $1550 \AA$, the N v 2s-2p transitions at 1238 and $1242 \AA$, and the $\mathrm{H}_{\mathrm{I}} \mathrm{Ly} \alpha$ line from the obscuring medium all have optical depths of the order of unity, so should produce detectable UV absorption lines. On the other hand, the optical depth in the $\mathrm{O} v$ $2 s-2 p$ lines is of the order of 100 . This shows that the predicted optical depth of the UV lines of the obscurer is very sensitive to the parameters of this obscuring medium. Unfortunately, in August 2016 no UV spectra of NGC 3783 were taken that could validate our model.

\subsection{Obscuration at other epochs}

We have also investigated possible obscuration using observations from other satellites. The spectra taken by BeppoSAX in June 1998 (De Rosa et al. 2002) and Suzaku in July 2009 (Reis et al. 2012) do not show apparent evidence for obscuration. However the ASCA spectra taken in 1993, when compared to later data taken in 1996, show evidence for strong spectral differences in the soft X-ray band (George et al. 1998). These were interpreted by the authors as corresponding to absorbing gas with more than one component, where one absorbing component with a typical column density of $10^{25} \mathrm{~m}^{-2}$ that was present in 1993 would have moved out of the line of sight in 1996. While the spectral resolution of the ASCA SIS detectors in the soft $\mathrm{X}$-ray band hampers a detailed study like is possible with the XMM-Newton data, the basic conclusions of George et al. (1998) are consistent with a scenario where in 1993 moderate obscuration is present.

This is confirmed by our own fits to these data. The number of free parameters of our model is very small: only the power law and comptonised soft component parameters, the column density, and covering factor of the obscurer are left free in the fit. The warm absorber is completely determined by its column densities and ionisation state as derived from the grating spectra taken in 2000 and 2001, adjusted for the contemporaneous ionising SED. The column density and covering factor we derive for the 1993 spectrum are remarkably similar to those on August 22, 2016, with a two-times higher continuum flux in 1993. 


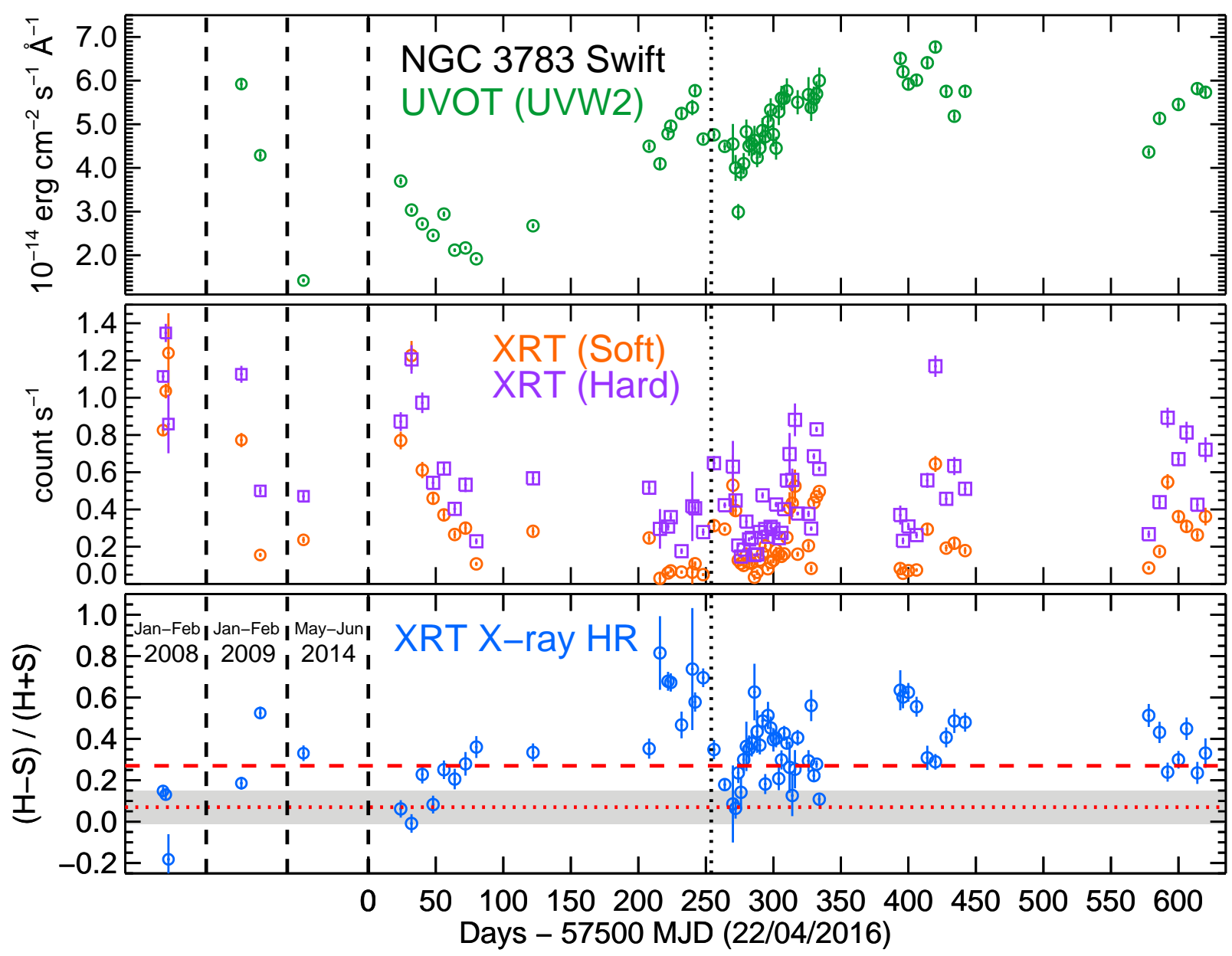

Fig. 4. Swift light curves in different bands. The vertical dotted line indicates 1 January 2017. The data have been averaged over two days. The last datapoint was taken on January 3, 2018. Top panel: UVOT flux; middle panel: soft (0.3-1.5 keV) and hard (1.5-10 keV) X-ray count rate; bottom panel: hardness ratio $R=(H-S) /(H+S)$ with $S$ and $H$ the soft and hard X-ray count rates. Dotted horizontal line: average hardness without obscuration as described in the text; the grey area corresponds, with its typical variations, to variations in the continuum. Dashed horizontal line: hardness above which there is strong obscuration (see text).

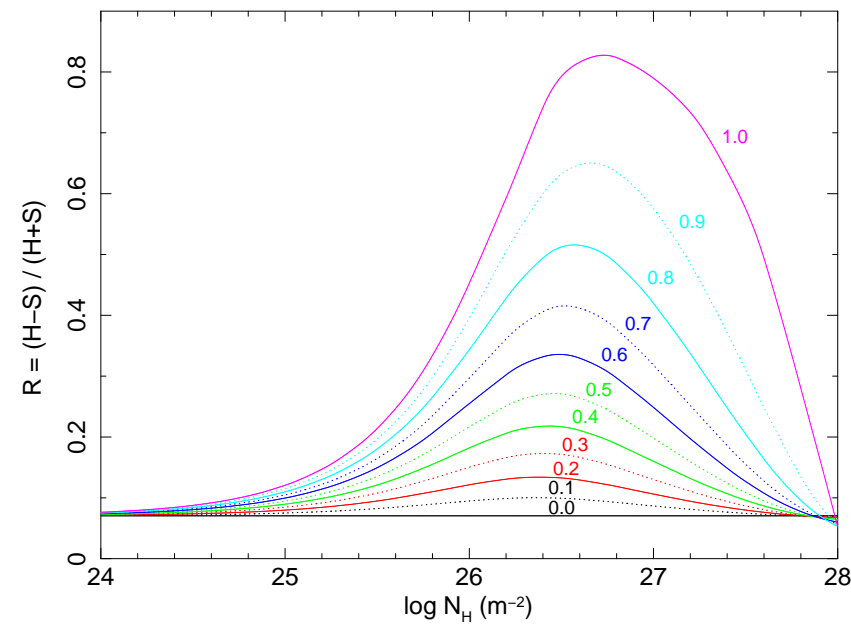

Fig. 5. Predicted Swift hardness ratio $R$ as a function of the obscuring column density $N_{\mathrm{H}}$ (curves) and the covering factor $C_{\mathrm{f}}$ (labels).

While our model for the ASCA data matches the broad-band dip in the spectrum well, the fit is not perfect and shows a few systematic deviations at the $10-20 \%$ level near $0.65 \mathrm{keV}(20 \AA)$ and $1 \mathrm{keV}$. These deviations are much larger than the nomi-

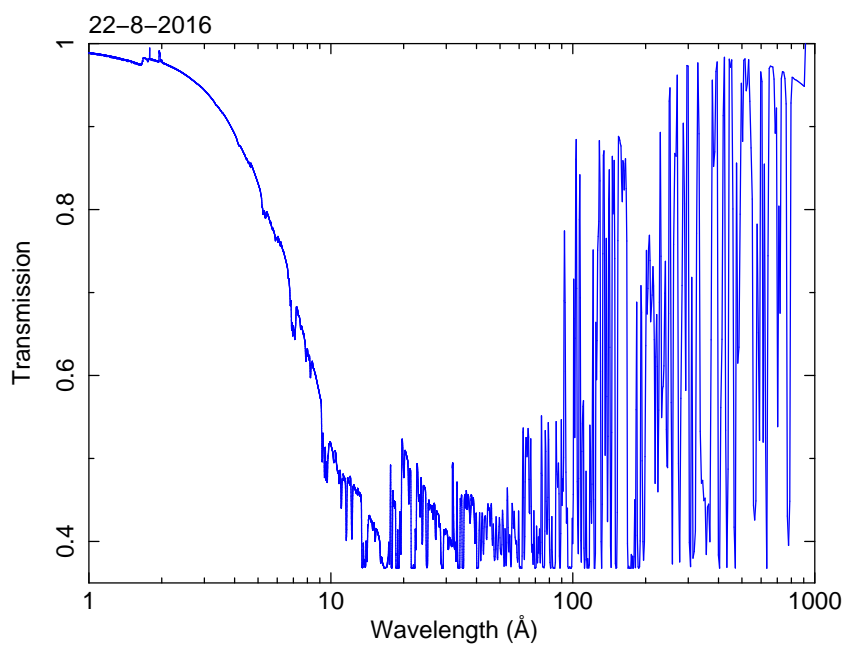

Fig. 6. Transmission of the obscurer on 22 August 2016.

nal statistical uncertainties on the data in this energy band (see Fig. 3). They might be caused by a combination of various processes, like our constraint on the UV flux, secular changes of some components over the 23 years between 1993 and 2016, or calibration issues, and so on. The relatively low spectral 
resolution of ASCA in the soft X-ray band prohibits us from definitively accounting for these deviations. However, it is surprising that with so few free parameters we can still obtain a rough fit that catches the main obscuration trough. In any case, both our analysis and the original modeling by George et al. (1998) indicate the presence of additional obscuring material in the 1993 ASCA spectrum.

Finally, we note that the spectrum taken in 2000 has the lowest column density of all three ASCA spectra, with only $3.6 \times 10^{25} \mathrm{~m}^{-2}$ (Table 2). This is only $20 \%$ of the column density of the distant warm absorber for the same ionisation parameter, and therefore, despite its nominal statistical uncertainty, we do not claim that there is obscuration in this spectrum. The systematic uncertainties are likely the largest for this spectrum, with the highest ratio of the $2-10 \mathrm{keV}$ power law flux to the comptonised component flux of all spectra (see Table 2). This is also consistent with our assumption that the XMM-Newton and Chandra spectra taken in 2000 and 2001, the same epoch as the ASCA spectrum, were free of obscuration. More observations are needed to confirm whether the apparent anti-correlation between power-law luminosity and column density of the obscuring material (see Table 2) is real or is a chance occurrence.

\subsection{The frequency of obscuration}

Obscuration appears to be more frequent in NGC 3783 than previously thought. About half of all Swift observations show evidence for moderate to strong obscuration, and also in the more distant past, obscuration events have occurred like those seen with ASCA in 1993 and the three RXTE events mentioned in the introduction; see Markowitz et al. (2014).

In fact, the sparsity of most data makes it hard to define an obscuration event. The densely sampled part of the Swift light curve in spring 2017 shows strong variability, with almost always some level of obscuration present. It is a matter of semantics whether one uses the term "event" to refer to just the peaks of this obscuration or the entire period of a few months containing these peaks.

Interestingly, our modelling indicates that even the ASCA 1996 spectrum may show some obscuration, albeit with a low column density of $\sim 10^{26} \mathrm{~m}^{-2}$ and only $27 \%$ covering factor. Such values may also occur during the "softest" intervals of longer-lasting obscuration periods as seen by Swift. In most other spectral analyses, such a parameter range of the obscuring gas may have been easily overlooked. The continuum obscuration may be compensated by modifying the shape or relative intensity of other spectral parameters like the main power law, the soft excess, or the reflection component.

In the cases that we studied, obscuration could be proven using the available high-resolution X-ray spectra combined with broad-band SED information. Whenever high-resolution UV spectra are also available, like for NGC 5548 (Kaastra et al. 2014b) or the December 2016 observations of NGC 3783 (Mehdipour et al. 2017), the dynamical state of the obscuring stream can be determined from the UV line profiles. Also, the observed UV lines set bounds on the allowed range of ionisation parameter for the obscuring gas, something that cannot be done in the X-rays in these cases due to the lack of spectral features.

The higher frequency of obscuration events that we find here also implies that for the distant emitting gas of the ionisation cones or narrow line region, the effective ionising continuum radiation, as averaged over several years for gas at parsec distance scales, may be smaller than if one were to

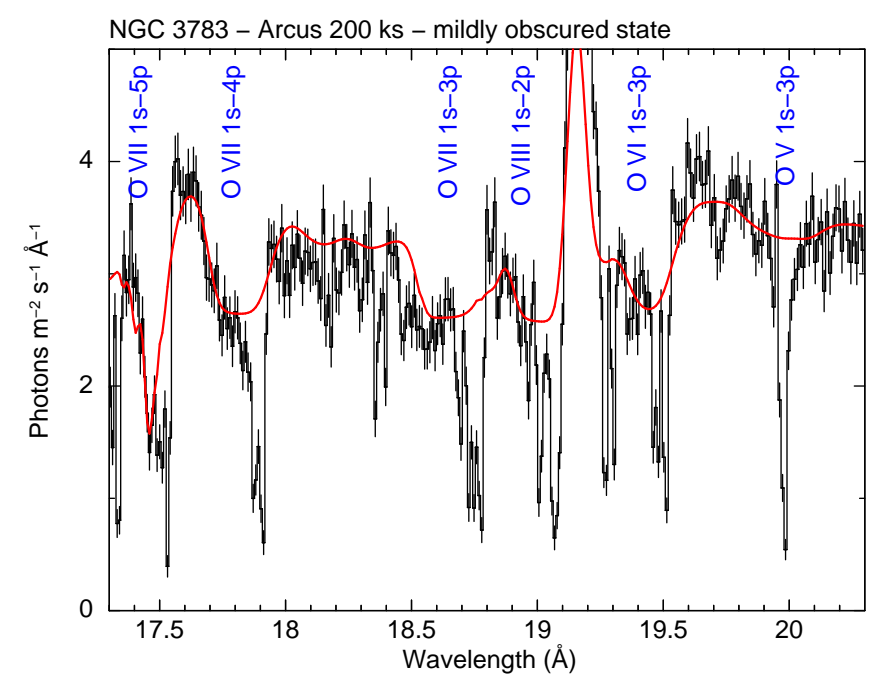

Fig. 7. Part of a simulated spectrum for the Arcus mission for NGC 3783 with the same source parameters as it showed on 22 August 2016 (this paper). The simulated spectrum is shown as the black histogram with error bars. The red curve shows the obscured continuum that the warm absorber receives, scaled down by a factor of 3.3. The narrow $\mathrm{O}$ VIII Lyman alpha emission line at $19.15 \AA$ is from a remote region and is cutoff in this plot. This figure shows that Arcus will be able to separate the narrow absorption lines of the warm absorber from the broad absorption lines from the obscurer.

adopt the contemporaneous SED of an unobscured epoch. This will affect the modelling of the photoionised gas in those regions.

Finally, as can be deduced from the light curves, the obscuration is highly variable in terms of column density and covering factor. Similar conclusions have been reached for the obscuration in NGC 5548. The obscuring streams apparently have relatively large density and therefore short recombination times, hence adjust their ionisation state quickly to changes of the ionising continuum. The strong variability may also be caused by a rather inhomogeneous and irregular matter distribution within the outflow, causing rapid column density and covering factor variations.

\subsection{Future prospects}

A more detailed study of obscuration effects, and in a larger sample, requires monitoring with multiple instruments. At this moment, we need X-ray grating spectra to characterise the X-ray obscuration, and in addition high-resolution UV spectra to confirm (like in NGC 5548, Kaastra et al. 2014b) or measure (like in NGC 3783, Mehdipour et al. 2017) the ionisation parameter of the outflow. Ultraviolet spectra are also needed to measure the outflow velocity of the obscurer. Moreover, because the obscuration is highly variable and transient, it requires either extensive monitoring or triggered observations.

Proposed new X-ray instrumentation may offer another solution. The Arcus mission (Smith et al. 2016) has an order-ofmagnitude higher effective area and spectral resolution compared with the currently most sensitive spectrometer, the RGS on board of XMM-Newton. This is sufficient to separate the narrow absorption lines of the warm absorber from the broader absorption lines of the obscurer in the X-ray band, which is impossible with our present instrumentation; see Fig. 7 for a simulation of the August 22, 2016, spectrum of NGC 3783. 
This does not imply that we do not need high-resolution UV spectra in addition. The combination of high-resolution X-ray and UV spectra would allow us to find the covering factors in both bands individually and would also allow for much better constraint of the geometry of the inner parts of the AGN.

\section{Conclusions}

By analysing archival Chandra HETGS spectra of NGC 3783, we have found that also in August 2016 obscuration of the nucleus took place, albeit with a lower column density than four months later in December 2016. We have also identified an epoch when ASCA is likely to have caught the source in a mildly obscured state. Swift monitoring indicates that obscuration can be seen in a significant fraction of all observations. Obscuring column densities $>5 \times 10^{25} \mathrm{~m}^{-2}$ occur in more than $66 \%$ of all Swift observations. Obscuration of the nuclear radiation by gas at broad-line distances may be more common than we thought, at least in NGC 3783. To determine whether this holds for other $\mathrm{AGN}$ as well requires further study.

Acknowledgements. SRON is financially supported by NWO, The Netherlands Organization for Scientific Research. The research at the Technion is supported by the I-CORE program of the Planning and Budgeting Committee (grant number 1937/12). EB acknowledges funding from the European Union's Horizon 2020 research and innovation programme under the Marie Sklodowska-Curie grant agreement no. 655324. SB and MC acknowledge financial support from the Italian Space Agency under grant ASI-INAF I/037/12/0, and under the agreement ASI-INAF n.2017-14-H.O. BDM acknowledges support from Polish National Science Centre grant Polonez 2016/21/P/ST9/04025. POP aknowledges support from the CNES and French PNHE. GP acknowledges support by the Bundesministerium für Wirtschaft und Technologie/Deutsches Zentrum für Luft- und Raumfahrt (BMWI/DLR, FKZ 50 OR 1604) and the Max Planck Society.

\section{References}

Crenshaw, D. M., Kraemer, S. B., \& George, I. M. 2003, ARA\&A, 41, 117

De Rosa, A., Piro, L., Fiore, F., et al. 2002, A\&A, 387, 838

Ebrero, J., Kriss, G. A., Kaastra, J. S., \& Ely, J. C. 2016, A\&A, 586, A72

George, I. M., Turner, T. J., Mushotzky, R., Nandra, K., \& Netzer, H. 1998, ApJ, 503,174

Huenemoerder, D. P., Mitschang, A., Dewey, D., et al. 2011, AJ, 141, 129

Kaastra, J. S. 2017, A\&A, 605, A51

Kaastra, J. S., \& Bleeker, J. A. M. 2016, A\&A, 587, A151

Kaastra, J. S., Mewe, R., \& Nieuwenhuijzen, H. 1996, in UV and X-ray Spectroscopy of Astrophysical and Laboratory Plasmas, eds. K. Yamashita, \& T. Watanabe, 411

Kaastra, J. S., Ebrero, J., Arav, N., et al. 2014a, A\&A, 570, A73

Kaastra, J. S., Kriss, G. A., Cappi, M., et al. 2014b, Science, 345, 64

Longinotti, A. L., Krongold, Y., Kriss, G. A., et al. 2013, ApJ, 766, 104

Markowitz, A. G., Krumpe, M., \& Nikutta, R. 2014, MNRAS, 439, 1403

Mehdipour, M., Kaastra, J. S., Kriss, G. A., et al. 2017, A\&A, 607, A28

Reis, R. C., Fabian, A. C., Reynolds, C. S., et al. 2012, ApJ, 745, 93

Risaliti, G., Elvis, M., Fabbiano, G., et al. 2007, ApJ, 659, L111

Risaliti, G., Nardini, E., Salvati, M., et al. 2011, MNRAS, 410, 1027

Smith, R. K., Abraham, M. H., \& Allured, R. et al. 2016, in Space Telescopes and Instrumentation 2016: Ultraviolet to Gamma Ray, Proc. SPIE, 9905, 99054M

Turner, T. J., Nandra, K., Turcan, D., \& George, I. M. 2001, X-ray Astronomy: Stellar Endpoints, AGN, and the Diffuse X-ray Background, 599, 991 